\title{
The vascular occlusion test using multispectral imaging: a validation study
}

\section{The VASOIMAGE study}

\author{
Arnoud A. Bruins ${ }^{1,2}$ (D) Diederik G. P. J. Geboers ${ }^{1,2}$ - Jacob R. Bauer ${ }^{3}$. John H. G. M. Klaessens ${ }^{4}$. \\ Rudolf M. Verdaasdonk ${ }^{5}$. Christa Boer ${ }^{1,2}$
}

Received: 13 September 2019 / Accepted: 14 December 2019

C) Springer Nature B.V. 2020

\begin{abstract}
Multispectral imaging (MSI) is a new, non-invasive method to continuously measure oxygenation and microcirculatory perfusion, but has limitedly been validated in healthy volunteers. The present study aimed to validate the potential of multispectral imaging in the detection of microcirculatory perfusion disturbances during a vascular occlusion test (VOT). Two consecutive VOT's were performed on healthy volunteers and tissue oxygenation was measured with MSI and near-infrared spectroscopy (NIRS). Correlations between the rate of desaturation, recovery and the hyperemic area under the curve (AUC) measured by MSI and NIRS were calculated. Fifty-eight volunteers were included. The MSI oxygenation curves showed identifiable components of the VOT, including a desaturation and recovery slope and hyperemic area under the curve, similar to those measured with NIRS. The correlation between the rate of desaturation measured by MSI and NIRS was moderate: $r=0.42$ $(\mathrm{p}=0.001)$ for the first and $\mathrm{r}=0.41(\mathrm{p}=0.002)$ for the second test. Our results suggest that non-contact multispectral imaging is able to measure changes in regional oxygenation and deoxygenation during a vascular occlusion test in healthy volunteers. When compared to measurements with NIRS, correlation of results was moderate to weak, most likely reflecting differences in physiology of the regions of interest and measurement technique.
\end{abstract}

Keywords Deoxyhemoglobin $\cdot$ Multispectral imaging $\cdot$ Near-infrared spectroscopy $\cdot$ Vascular occlusion test

\section{Introduction}

Arnoud A. Bruins

a.bruins@amsterdamumc.nl

1 Departments of Anesthesiology, Amsterdam UMC, VU University, location VUmc, De Boelelaan 1117, 1081 HV Amsterdam, The Netherlands

2 Amsterdam Cardiovascular Sciences, De Boelelaan 1117, 1081 HV Amsterdam, The Netherlands

3 The Norwegian Colour and Visual Computing Laboratory, Norwegian University of Science and Technology (NTNU), Gjøvik, Norway

4 Department of Clinical Physics, Medical Center Leeuwarden, Leeuwarden, The Netherlands

5 TechMed Center, BioMedical Photonics \& Medical Imaging, University of Twente, Enschede, The Netherlands
The microcirculation, consisting of the smaller arteries, arterioles, capillaries and venules, has a crucial role in the process of tissue oxygenation, perfusion and nutritional exchange [1-3]. Adequate microvascular perfusion is therefore indispensable for normal organ function. Previous research has indicated that macrohemodynamic parameters, such as the mean arterial pressure, do not represent microcirculatory perfusion in the perioperative setting [4-7].

Measurements of microcirculatory behavior in the clinical setting present a particular challenge, because vessel structure is inhomogeneous and perfusion is highly variable, especially in situations with major impact on system hemodynamics such as anesthesia and surgery. A number of devices, for example near-infrared spectroscopy (NIRS), have been developed to measure variables as a surrogate for the microcirculatory condition [8]. NIRS can be used 
to measure tissue oxygen saturation $\left(\mathrm{rSO}_{2}\right)$ and is also able to evaluate dynamic changes in oxygen saturation. During a vascular occlusion test, dynamic changes expressed in a desaturation slope, measured with NIRS, represent tissue oxygen extraction. The hyperemic phase after reperfusion reflects local capillary recruitment. Changes to those parameters are related to the functional activity of the microcirculation [9-11]. For research purposes, NIRS has been used extensively. However, one its limitations for the clinical perioperative setting is the need for direct contact to the skin.

Multispectral imaging (MSI) however is an emerging, non-invasive, non-contact imaging modality that has the potential to provide real-time information about regional tissue oxygenation as a surrogate for microcirculatory perfusion. MSI works by collecting data across the visible bands of the electromagnetic spectrum in order to deliver a unique reflectance spectrum for each pixel in the image [12, 13].

So far MSI has been used for for research purposes in animals and humans in haemorrhagic shock, diabetic ulcers and resuscitation [14-19]. However, MSI has limited been validated.

The primary aim of this validation study was to assess the ability of the multispectral camera in detecting regional tissue desaturation and recovery during a vascular occlusion test in healthy volunteers. Furthermore, oxyhemoglobin and deoxyhemoglobin levels measured with multispectral imaging and the $\mathrm{rSO}_{2}$ measured by near-infrared spectroscopy were compared.

\section{Methods}

\subsection{Study design}

This open label, non-randomized observational study was approved by the Human Subjects Committee of Amsterdam UMC, location VU University Medical Center (METc16.315). Written informed consent was obtained from all volunteers prior to participation.

All volunteers between 18 and 65 years old, from all ethnicities were eligible for participation in the study. Volunteers with a history of vascular disease (i.e. Raynaud's disease, peripheral vascular dysfunction or peripheral artery disease), diabetes mellitus, infectious diseases or skin anomalies of the forearm (i.e. burn wounds or large scars) were excluded from participation. The initial blood pressure was measured on both arms, volunteers with more than $10 \mathrm{mmHg}$ difference in systolic blood pressure between both arms were excluded.

\subsection{Measurements}

Demographic data, baseline blood pressure, heart rate and temperature were collected. Volunteers were seated in a quiet environment with a constant room temperature of $21^{\circ} \mathrm{C}$. A blood pressure cuff was placed around the left upper forearm of the volunteer and inflated until $30 \mathrm{mmHg}$ above the initial systolic blood pressure. The cuff remained inflated until regional oxygen saturation $\left(\mathrm{rSO}_{2}\right)$ was decreased to $40 \%$ as measured with near-infrared spectroscopy (NIRS).

The vascular occlusion test was based on the report of Mayeur et al. that stated that cuff inflation for the time period required to reduce oxygen to $40 \%$ is more appropriate than a fixed time of cuff inflation [20]. When a saturation of $40 \%$ was reached, the blood pressure cuff was deflated rapidly.

Baseline was defined when a steady state was reached, measured with NIRS. After reaching baseline the vascular occlusion test started. The desaturation slope (des $\mathrm{rSO}_{2}$, percentage per second) was defined as the slope of the desaturation curve starting at baseline and ending at the lowest point of the curve, measured with NIRS. The recovery slope (rec $\mathrm{rSO}_{2}$, percentage per second) was defined as the slope of the curve starting at the lowest point of tissue oxygenation until return to baseline, measured with NIRS. The desaturation slope (des $\mathrm{StO}_{2}$, arbitrary units) measured with MSI. The recovery slope (recStO ${ }_{2}$, arbitrary units) measured with MSI. Finally, the hyperemic area under the curve was calculated from the area under the curve from the point where the recovery slope intersects the baseline until the curve returns to baseline after the hyperemic phase.

Measurements were performed using the MSI and NIRS from before inflation of the pneumatic cuff until recovery of the saturation to baseline levels [20] (Fig. 1). After a resting period of $10 \mathrm{~min}$ the test was repeated to determine intrasubject variability.

\subsection{Determination of $\mathrm{NIRS}_{\mathrm{rSO}_{2}}$}

Regional tissue oxygen saturation was measured using a sensor (Somasensor SAFB-SM, Covidien, Mansfield, USA) at the thenar eminence of the hand using the NIRS (INVOS 5100C-PA; Covidien, Zaltbommel, the Netherlands) oxygenation monitor with a 4-6 s interval.

\subsection{Determination of MSI oxyhemoglobin and deoxyhemoglobin}

Spectral images of visual light between 400 and $600 \mathrm{~nm}$, most prominent for oxyhemoglobin, were collected using a spectral filter array camera (xiQ, MQ022HG-IM-SM4X4 VIS, XIMEA Münster, Germany). Each pixel containing a 


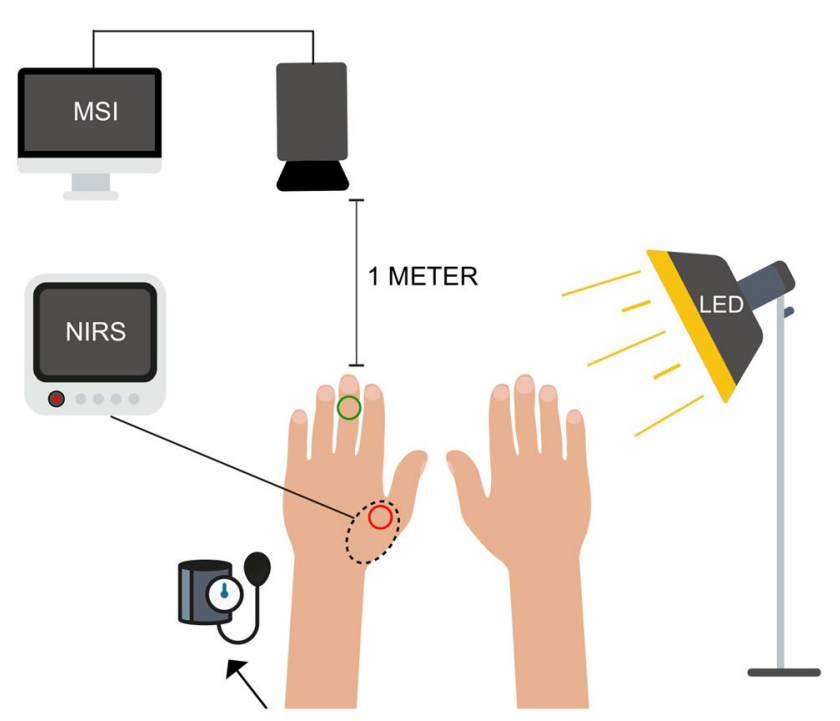

Fig. 1 Test setup during vascular occlusion test. Hands of the volunteer resting on a table. Multispectral camera (xiQ, MQ022HGIM-SM4X4 VIS, XIMEA Münster, Germany) placed $1 \mathrm{~m}$ above the subjects' hands. Two regions of interest were chosen (green and red circle). Parallel measurements with NIRS at the thenar eminence (INVOS 5100C-PA; Covidien, Zaltbommel, the Netherlands). Light source was placed on a $45^{\circ}$ angle. Dataprocessing was performed on an external computer. $M S I$ multispectral imaging, NIRS near-infrared spectroscopy

spectrum along these wavelengths. These spectra include the absorption peaks for oxyhemoglobin and deoxyhemoglobin (oxyHb and deoxyHb) in the visual range. Using a spectral calibration, spatial processing, spectral correction and three key wavelengths for oxygenation estimation. Gray scale images were generated in which the brightness of each pixel is related to the $\mathrm{StO}_{2}$ at the given location. A region of interest in proximity to the reference region of interest was chosen for average measurements (Fig. 1). A bright white LED panel attached to a Manfrotto-arm was used for uniform illumination in a $45^{\circ}$ angle to avoid specular reflections.

\subsection{Statistical analysis}

Data were analyzed using Graphpad Prism 7 (Graphpad Software Inc., San Diego, California) and SPSS Statistics version 22.0 (IBM, New York, USA). All included subjects were incorporated in the statistical analysis. Descriptive statistics include mean, median, standard deviation, standard error of the mean and the interquartile range. To compare parametric data a paired sample t test was used. A p-value of $<0.05$ was considered significant.

Power analysis was conducted for an effect size of $r=0.40$, with an alpha error of 0.05 and a power of 0.80 , which resulted in a minimal desired sample size of 47 volunteers.
Table 1 General characteristics of the study group $(n=58)$

\begin{tabular}{ll}
\hline Variables & Values \\
\hline Age (years) & $29 \pm 9$ \\
Gender (male:female) & $26: 32$ \\
Weight $(\mathrm{kg})$ & $73 \pm 13$ \\
Height $(\mathrm{cm})$ & $178 \pm 9$ \\
BMI $\left(\mathrm{kg} / \mathrm{m}^{2}\right)$ & $23 \pm 3$ \\
Systolic blood pressure left $(\mathrm{mmHg})$ & $119 \pm 9$ \\
Diastolic blood pressure left $(\mathrm{mmHg})$ & $76 \pm 7$ \\
Systolic blood pressure right $(\mathrm{mmHg})$ & $120 \pm 10$ \\
Diastolic blood pressure right $(\mathrm{mmHg})$ & $76 \pm 6$ \\
HR (beats/min) & $68 \pm 5$ \\
Temperature $\left({ }^{\circ} \mathrm{C}\right)$ & $36.5 \pm 0.3$ \\
\hline
\end{tabular}

Data are represented as absolute values, means \pm SD

$B M I$ body mass index, $H R$ heart rate

The association between the $\mathrm{MSI} \mathrm{StO}_{2}$ and NIRS $\mathrm{rSO}_{2}$ recovery slope was analyzed using Pearson correlation coefficients. The level of agreement between the MSI and NIRS $\mathrm{rSO}_{2}$ recovery slope was analyzed by intraclass correlation coefficients.

\section{Results}

\subsection{Volunteer demographics and test characteristics}

Two vascular occlusion tests were performed in 58 volunteers, and demographic and baseline characteristics are presented in Table 1. One case was excluded due to erroneous NIRS measurements during the vascular occlusion phase in the second test. The mean duration of the VOT was $81 \pm 27 \mathrm{~s}$ for the first and $92 \pm 27 \mathrm{~s}$ for the second test. The time from reperfusion until return to baseline of the $\mathrm{rSO}_{2}$ measured by NIRS was $121 \pm 39 \mathrm{~s}$ for the first and $145 \pm 53 \mathrm{~s}$ for the second test. Values of desaturation slopes, recovery slopes and hyperemic area under the curves for the NIRS and MSI measurements can be found in Tables 2 and 3, respectively.

In $16 \%$ (19 out of 117 cases) the tissue oxygenation curve measured with the multispectral camera did not demonstrate a hyperemic phase, whereas the same cases did have a clear hyperemic phase in the NIRS measurements.

\subsection{Curve form}

The MSI StO $\mathrm{O}_{2}$ curves showed identifiable components of the VOT i.e. a desaturation and recovery slope and hyperemic area under the curve as compared to NIRS. Figure 2 shows a typical example of a $\mathrm{MSI} \mathrm{StO}_{2}$ curve. 
Table 2 Tissue oxygenation at the thenar eminence during a vascular occlusion test in healthy volunteers measured with near infrared spectroscopy

\begin{tabular}{|c|c|c|c|}
\hline Variables & NIRS VOT1 & NIRS VOT2 & P-value \\
\hline Baseline $\mathrm{rSO}_{2}(\%)$ & $63.5 \pm 7.8$ & $64.3 \pm 8.3$ & 0.32 \\
\hline $\begin{array}{l}\text { Duration of vascular occlu- } \\
\text { sion (s) }\end{array}$ & $81 \pm 27$ & $92 \pm 27$ & $<0.01$ \\
\hline Minimal $\mathrm{rSO}_{2}(\%)$ & $38.8 \pm 1.5$ & $39.5 \pm 1.0$ & $<0.01$ \\
\hline Maximal $\mathrm{rSO}_{2}(\%)$ & $82.1 \pm 10.7$ & $86.9 \pm 8.7$ & $<0.01$ \\
\hline Reperfusion time (s) & $120.5 \pm 539.4$ & $145.0 \pm 53.0$ & 0.03 \\
\hline $\begin{array}{l}\text { Des } \mathrm{rSO}_{2} \text { desaturation slope } \\
(\% / \mathrm{s})\end{array}$ & $-0.34 \pm 0.09$ & $-0.31 \pm 0.1$ & $<0.01$ \\
\hline $\begin{array}{l}\text { Rec } \mathrm{rSO}_{2} \text { recovery slope } \\
(\% / \mathrm{s})\end{array}$ & $2.4 \pm 1.1$ & $2.5 \pm 0.9$ & 0.47 \\
\hline $\begin{array}{l}\text { Hyperemic AUC (arbitrary } \\
\text { units) }\end{array}$ & $1144 \pm 625$ & $1657 \pm 748$ & $<0.01$ \\
\hline
\end{tabular}

Data are presented as mean $\pm \mathrm{SD}$. The duration of vascular occlusion is defined as the duration of cuff inflation. The reperfusion time is defined as the duration of cuff deflation until return of $\mathrm{rSO}_{2}$ to baseline

NIRS near infrared spectroscopy, VOT vascular occlusion test, $r \mathrm{SO}_{2}$ regional tissue oxygen saturation. Des $r \mathrm{SO}_{2}$ desaturation slope $\mathrm{rSO}_{2}$, Rec $r \mathrm{SO}_{2}$ recovery slope $\mathrm{rSO}_{2}, A U C$ area under the curve

\subsection{Correlation coefficients}

There was a moderate correlation between desaturation slopes measured by NIRS and MSI for the first $(r=0.42$; $p=0.001)$ and second test $(r=0.41 ; p=0.002)$, Fig. 3. There was no correlation between the recovery slopes for the first test $(\mathrm{r}=0.19 ; \mathrm{p}=0.16)$ and a weak correlation for the second test $(r=0.27 ; p=0.04)$. There was no correlation between the hyperemic AUC for the first $(\mathrm{r}=0.28 ; \mathrm{p}=0.06)$ and $\mathrm{a}$ moderate correlation for the second test $(r=0.41 ; p=0.004)$.

\subsection{Intrasubject variability}

The intra-subject variability between the slopes and hyperemic AUC measured by NIRS, during the first and the second test was moderate to good: 0.77 (95\% CI 0.56-0.87; $\mathrm{p}<0.001)$ for the desaturation slopes, $0.72(95 \% \mathrm{CI}$ $0.52-0.83 ; \mathrm{p}<0.001)$ for the recovery slopes and $0.61(95 \%$ CI $0.11-0.81 ; \mathrm{p}<0.001)$ for the hyperemic AUC.

The intra-subject variability between for MSI measurements was also moderate to good: 0.87 (95\% CI 0.77-0.92; $\mathrm{p}<0.001)$ for the desaturation slopes, $0.74(95 \% \mathrm{CI}$ $0.49-0.86 ; \mathrm{p}<0.001)$ for the recovery slopes and $0.95(95 \%$ CI $0.91-0.97 ; \mathrm{p}<0.001)$ for the hyperemic area under the curve.

\section{Discussion}

In this validation study, multispectral imaging (MSI) was used to determine changes in oxy- and deoxyhemoglobin during a vascular occlusion test in healthy volunteers. Changes in hemoglobin oxygenation were compared with measurements made by near-infrared spectroscopy. As expected, the MSI camera was able to establish the anticipated changes in tissue oxygenation during a vascular occlusion test in healthy volunteers, showing a clear desaturation curve upon occlusion, recovery curve upon cuff release and in most cases hyperemic area under the curve as a sign of local capillary recruitment.

When comparing MSI and NIRS, weak to moderate correlations were found, which could be attributable to physiological differences of the tissue and/or technical differences in measurement techniques. First of all, the INVOS system uses wavelengths in the near infrared spectrum (730 and $810 \mathrm{~nm}$ ) to measure hemoglobin oxygenation. Contrastingly, the Ximea multispectral camera uses wavelengths in the visible spectrum between 400 and $600 \mathrm{~nm}$. Tseng et al. measured oxy- and deoxyhemoglobin with different wavelengths (500-600 $\mathrm{nm}$ and 600-1000 $\mathrm{nm}$ range), and found shorter wavelengths had less penetration depth. Furthermore, they found differences in oxy- and deoxyhemoglobin concentrations in the upper and lower regions of interest [21]. Measuring tissue saturation at different depths could explain the absence of correlation between the MSI and NIRS recovery slopes, hyperemic AUC and the absence of hyperemia in
Table 3 Tissue oxygenation at the thenar eminence during a vascular occlusion test in healthy volunteers measured with multispectral imaging

\begin{tabular}{lllc}
\hline Variables & MSI VOT1 & MSI VOT2 & P-value \\
\hline Baseline $\mathrm{StO}_{2}$ (arbitrary units) & $2.0 \pm 0.00061$ & $2.0 \pm 0.00067$ & 0.23 \\
Minimal $\mathrm{StO}_{2}$ (arbitrary units) & $1.996 \pm 0.0020$ & $1.996 \pm 0.0022$ & 0.01 \\
Maximal $\mathrm{StO}_{2}$ (arbitrary units) & $2.002 \pm 0.0011$ & $2.002 \pm 0.0011$ & 0.80 \\
$\mathrm{DesStO}_{2}$ (arbitrary units) & $-0.001 \pm 0.00003$ & $<-0.001 \pm 0.00003$ & 0.3 \\
RecStO$_{2}$ (arbitrary units) & $0.0007 \pm 0,0005$ & $0.0009 \pm 0.0006$ & $<0.01$ \\
Hyperemic AUC (arbitrary units) & $3.5 \pm 21.5$ & $5.4 \pm 33.6$ & 0.3 \\
\hline
\end{tabular}

Data are presented as mean $\pm \mathrm{SD}$

MSI multispectral imaging, $\mathrm{VOT}$ vascular occlusion test, $\mathrm{StO}_{2}$ peripheral tissue oxygen saturation measured with MSI, DesStO $\mathrm{O}_{2}$ desaturation slope, $\mathrm{ReCStO}_{2}$ recovery slope, $\mathrm{AUC}$ area under the curve 
Fig. 2 Typical examples of changes in tissue oxyhemoglobin and deoxyhemoglobin during a vascular occlusion test (VOT) measured with multispectral imaging (MSI). a changes in deoxyhemoglobin measured with MSI. b Changes is oxyhemoglobin measured with MSI. Continuous line indicates raw data, dashed line indicates polynomial interpolated data. The squared dotted line indicates linear interpolated data. Dotted line is baseline. $\mathrm{StO}_{2}$ tissue oxygen saturation, desStO $\mathrm{O}_{2}$ desaturation slope, recStO$O_{2}$ recovery slope, $d t$ time to reach baseline $\mathrm{StO}_{2}$, VOT vascular occlusion test
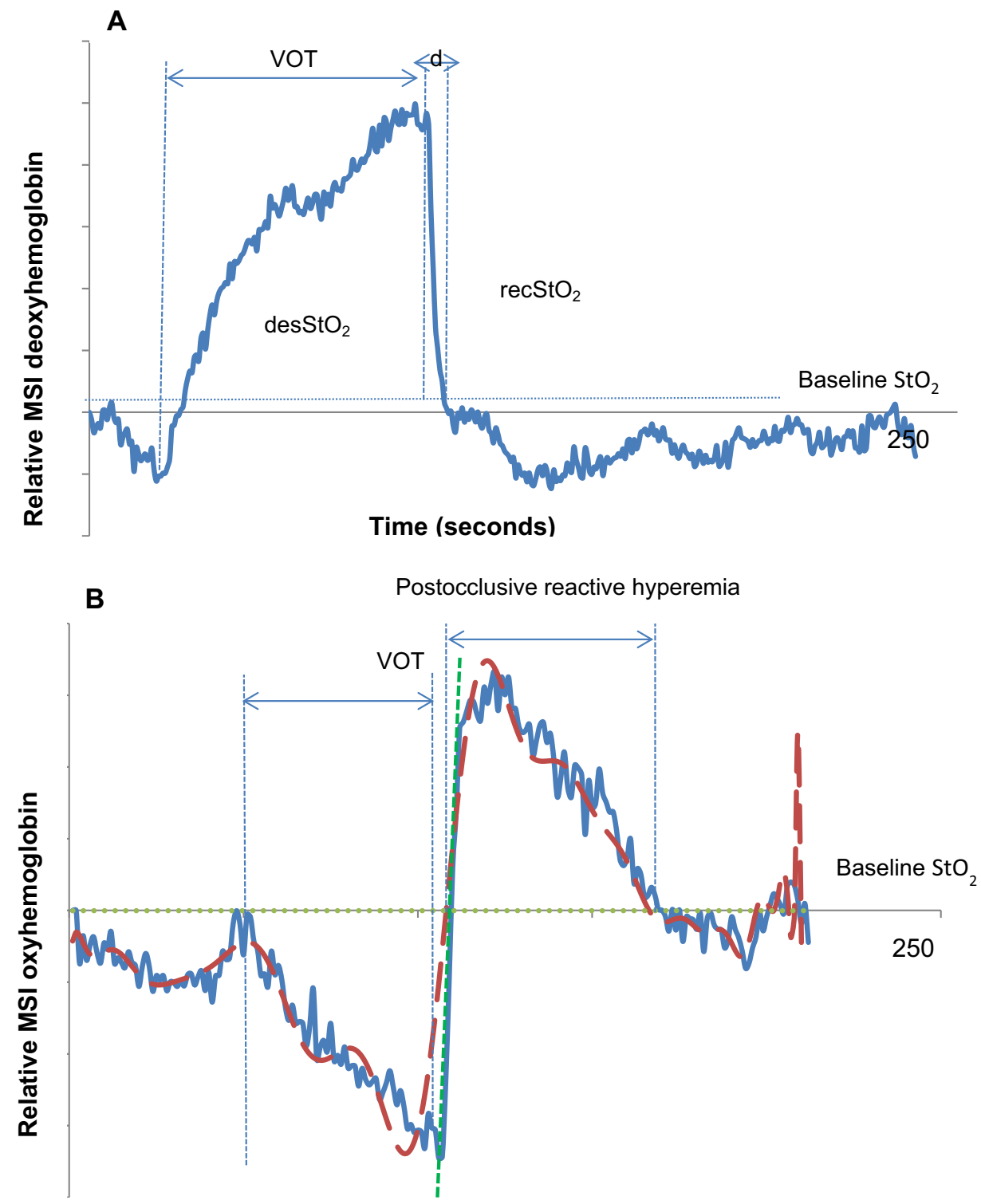

Time (seconds) part of the cases. Tissue measured by NIRS i.e. the deeper dermis and possibly underlying muscle tissue, is likely to have a higher oxygen metabolism than the upper dermis and epidermis measured by MSI [22].

The short duration of the reperfusion phase with relatively large changes in tissue saturation due to reperfusion, might have accentuated the differences in blood supply and metabolic demand of the more superficial versus deeper layers of the skin. Deeper tissues with relatively higher metabolic demand would desaturate more and thus elicit a greater hyperemic response.

Compared to previous work by Mayeur et al. in healthy volunteers undergoing a vascular occlusion test, the NIRS baseline $\mathrm{rSO}_{2}$ was lower in our study, the NIRS desaturation slopes found in this study declined faster, whereas the recovery slopes showed a less steep increase [20]. Mayeur et al. used comparable vascular occlusion protocols, but used an InSpectra near-infrared spectrometer, whereas in the current study the INVOS 5100C was used [20]. Although most commercially available near-infrared spectrometers use the same technology, significant differences in peripheral tissue oxygenation during vascular occlusion test and baseline values have been described [8, 23, 24]. These differences could be due to different number of wavelengths used in the sensors (i.e. two in the INVOS model versus four in the InSpectra model) and penetration depth variances according to wavelength and differences in computational algorithms. Additionally, $\mathrm{rSO}_{2}$ is dependent on the exact measurement 

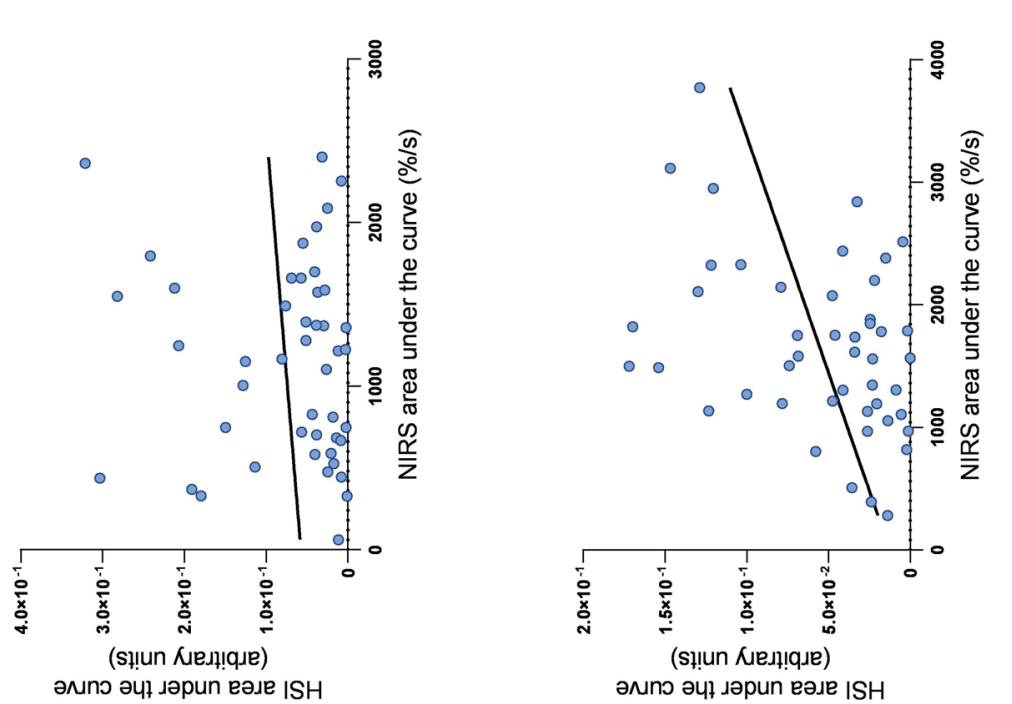

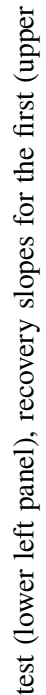
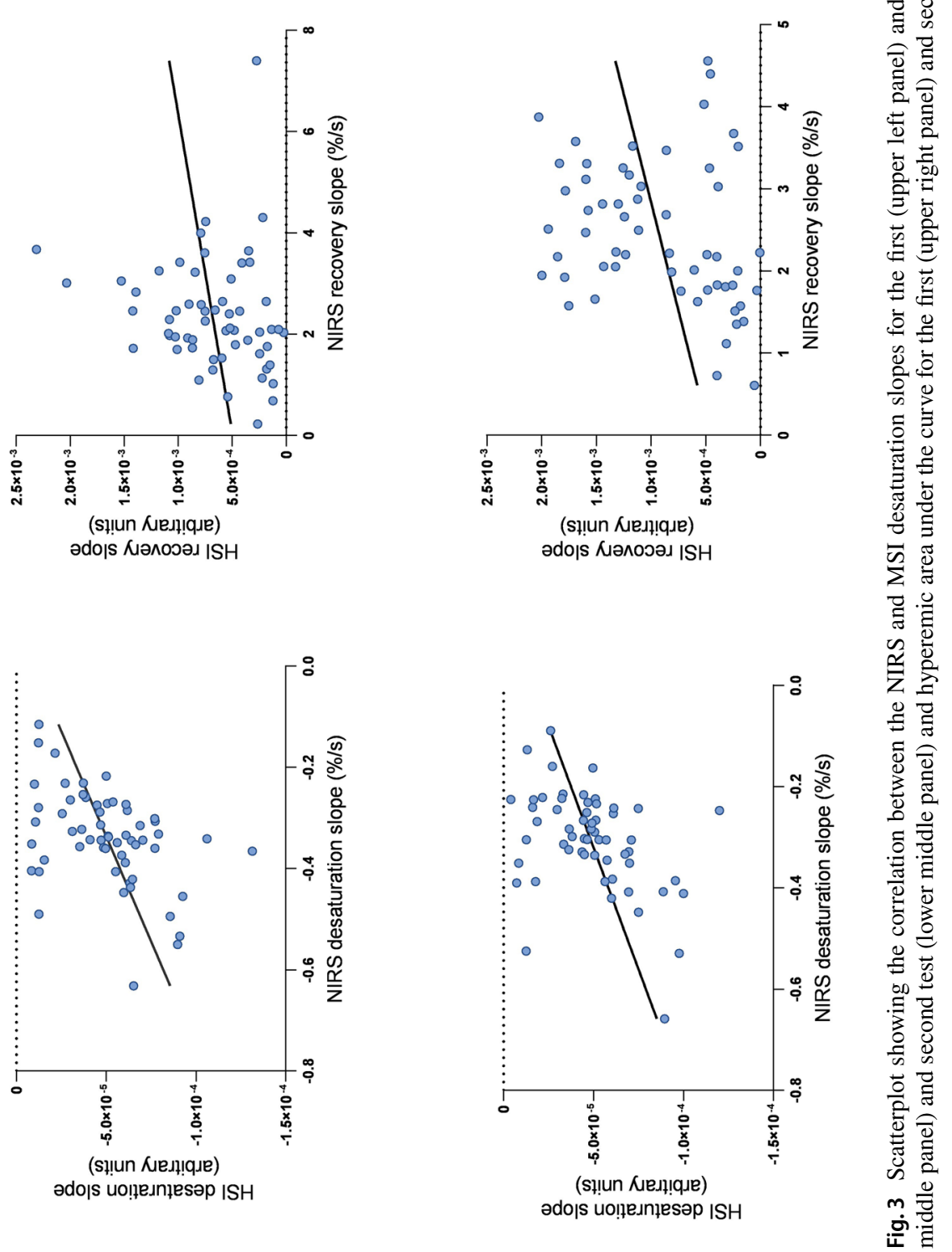
site, and intrasubject variation is observed with repeated measurements [25].

Moreover, technical differences and differences in acquisition frequency between NIRS and multispectral imaging could have had a negative influence on the correlation of the slopes and hyperemic AUC. The INVOS somatosensor uses a proximal and distal sensor, representing superficial (i.e. skin and bone) and deep tissue measurements. The superficial signals are subtracted and suppressed, whereas the multispectral camera measures the full thickness of the chosen region of interest mainly of the superficial tissues [26].

\subsection{Reproducibility of results}

The vascular occlusion test was repeated after a 10-min resting interval to determine reproducibility of results and intrasubject variations. Compared to the first test we found the second test to have a longer mean duration of vascular occlusion, greater maximal $\mathrm{rSO}_{2}$ after reperfusion, longer duration of reperfusion, lower desaturation slope values and higher AUC (Table 2). Tissue hypoxia caused by a transient vascular occlusion induces recruitment of closed microvessels and causes a hyperemic phase after release of the ischemic stimulus [27-30]. The differences mentioned above suggest persistent recruited capillaries and higher flow in the open microvessels, thus the 10-min resting interval seems to have been insufficient for normalization of tissue oxygenation. Recruitment of smaller vessels higher in dermis could have attributed to our finding a correlation between the NIRS and MSI recovery slopes and hyperemic area under the curve for the second, but not the first test.

Nevertheless, the intra-class correlation coefficients, when comparing the MSI slopes and hyperemic area under the curve of the first and second VOT in the same volunteer, were better overall than the ICC of the same measurements by NIRS. This suggests that reproducibility of MSI measurements was not inferior and possibly even better than those measured by NIRS.

\subsection{Study limitations}

For this study we used the INVOS spectrometer with a minimum measuring interval of 4-6 s, which provided two challenges. First of all, in previous literature where tissue oxygenation was measured with a NIRS device during a VOT, a biphasic course of the desaturation slope was described, with more variation across subjects in the latter, less steep part of the slope, reflecting differences in metabolic demand [31]. Using the initial $25 \%$ of the desaturation slope may minimize the effects of variations in metabolic demand among volunteers. Due to the relatively long measurement interval of the INVOS spectrometer however, we did not have enough data points for accurate linear regression using only the first $25 \%$ of the desaturation slope. Similarly, due the relatively long measurement interval and the short duration of the recovery slope, the point where the $\mathrm{rSO}_{2}$ returned to baseline had to be interpolated using linear regression, with few data points available.

\subsection{Future clinical feasibility of MSI}

A major advantage of MSI over NIRS is the non-contact nature of the device, in future research this would enable its use in monitoring visceral $\mathrm{StO} 2$ during sterile procedures. Furthermore, the small camera would allow use during (laparoscopic) surgery and at the bedside. In this study however, the amount of light was standardized by using a calibrated LED source and a room obscured from infiltrating light sources. Furthermore, the object of interest i.e. the hands, remained still during the entire experiment. For realtime $\mathrm{StO}_{2}$ monitoring in the perioperative setting software would have to be developed for processing the raw images and arbitrary units to $\mathrm{StO}_{2}$ values. Furthermore, the camera would have to be able to account for background and scattered light.

Moreover, $\mathrm{rSO}_{2}$ determination using NIRS is measurement site dependent. Bezemer et. al. found significant differences when comparing $\mathrm{rSO}_{2}$ changes during a vascular occlusion test with a probe on the forearm versus the thenar eminence [32]. Potential mechanisms for measurement site differences could be due to variations in composition of dermal and subdermal tissues (i.e. vessels or muscles), oxygen consumption and density of vascular plexus. Therefore, regional tissue saturation measured by NIRS (during a vascular occlusion test) varies with the chosen region of interest. In contrast, MSI provides spectral data for the entire depicted image and multiple regions of interest can be selected, thus facilitating identification of regions at risk for hypoxemia.

\section{Conclusion}

Our results suggest that non-contact multispectral imaging is able to measure changes in regional oxygenation and deoxygenation during a vascular occlusion test in healthy volunteers. When compared to measurements with NIRS, correlation of results was moderate to weak, most likely reflecting differences in physiology of the regions of interest and measurement technique.

Author contributions $\mathrm{AB}$ - study design, data collection, data analysis, interpretation of data, drafting manuscript, final approval. DG-data collection, data analysis, interpretation of data, drafting manuscript, final approval. JB - data collection, data analysis, interpretation of data, revising manuscript, final approval. JK-data collection, interpretation 
of data, revising manuscript, final approval. RV—-study design, interpretation of data, revising manuscript, final approval. CB-study design, interpretation of data, revising manuscript, final approval.

Funding This research did not receive any specific Grant from funding agencies in the public, commercial, or not-for-profit sectors.

\section{Compliance with ethical standards}

Conflict of interest The authors have no conflict of interest to declare.

Ethical approval This investigation involved Human Participants. The study was approved by the Human Subjects Committees of the VU Medical Center (METc 2016.315/NL56684.029.16). All participants provided written informed consent.

\section{References}

1. Lal C, Leahy MJ. An updated review of methods and advancements in microvascular blood flow imaging. Microcirculation. 2016;23(5):345-63.

2. Eriksson S, Nilsson J, Sturesson C. Non-invasive imaging of microcirculation: a technology review. Med Devices (Auckl). 2014;7:445-52.

3. Segal SS. Regulation of blood flow in the microcirculation. Microcirculation. 2005;12(1):33-45.

4. Stens J, de Wolf SP, van der Zwan RJ, Koning NJ, Dekker NA, Hering JP, et al. Microcirculatory perfusion during different perioperative hemodynamic strategies. Microcirculation. 2015;22(4):267-75.

5. Koning NJ, Atasever B, Vonk AB, Boer C. Changes in microcirculatory perfusion and oxygenation during cardiac surgery with or without cardiopulmonary bypass. J Cardiothorac Vasc Anesth. 2014;28(5):1331-400.

6. Koning NJ, Vonk AB, van Barneveld LJ, Beishuizen A, Atasever $\mathrm{B}$, van den Brom CE, et al. Pulsatile flow during cardiopulmonary bypass preserves postoperative microcirculatory perfusion irrespective of systemic hemodynamics. J Appl Physiol. 2012;112(10): 1727-1734.

7. Jhanji S, Lee C, Watson D, Hinds C, Pearse RM. Microvascular flow and tissue oxygenation after major abdominal surgery: association with post-operative complications. Intensive Care Med. 2009;35(4):671-7.

8. Lee JH, Park YH, Kim HS, Kim JT. Comparison of two devices using near-infrared spectroscopy for the measurement of tissue oxygenation during a vascular occlusion test in healthy volunteers (INVOS(R) vs InSpectra). J Clin Monit Comput. 2015;29(2):271-8

9. Lipcsey M, Woinarski NC, Bellomo R. Near infrared spectroscopy (NIRS) of the thenar eminence in anesthesia and intensive care. Ann Intensive Care. 2012;2(1):11.

10. Abdelmalak BB, Cata JP, Bonilla A, You J, Kopyeva T, Vogel $\mathrm{JD}$, et al. Intraoperative tissue oxygenation and postoperative outcomes after major non-cardiac surgery: an observational study. $\mathrm{Br}$ J Anaesth. 2013;110(2):241-9.

11. Bernet C, Desebbe O, Bordon S, Lacroix C, Rosamel P, Farhat $\mathrm{F}$, et al. The impact of induction of general anesthesia and a vascular occlusion test on tissue oxygen saturation derived parameters in high-risk surgical patients. J Clin Monit Comput. 2011;25(4):237-44.

12. Lu G, Fei B. Medical hyperspectral imaging: a review. J Biomed Opt. 2014;19(1):10901.
13. Muselimyan N, Swift LM, Asfour H, Chahbazian T, Mazhari $\mathrm{R}$, Mercader MA, et al. Seeing the invisible: revealing atrial ablation lesions using hyperspectral imaging approach. PLoS ONE. 2016;11(12):e0167760.

14. Cancio LC, Batchinsky AI, Mansfield JR, Panasyuk S, Hetz K, Martini D, et al. Hyperspectral imaging: a new approach to the diagnosis of hemorrhagic shock. J Trauma. 2006;60(5):1087-95.

15. Gillies R, Freeman JE, Cancio LC, Brand D, Hopmeier M, Mansfield JR. Systemic effects of shock and resuscitation monitored by visible hyperspectral imaging. Diabetes Technol Ther. 2003;5(5):847-55.

16. Greenman RL, Panasyuk S, Wang X, Lyons TE, Dinh T, Longoria L, et al. Early changes in the skin microcirculation and muscle metabolism of the diabetic foot. The Lancet. 2005;366(9498):1711-7.

17. Jeffcoate WJ, Clark DJ, Savic N, Rodmell PI, Hinchliffe RJ, Musgrove A, et al. Use of HSI to measure oxygen saturation in the lower limb and its correlation with healing of foot ulcers in diabetes. Diabet Med. 2015;32(6):798-802.

18. Khaodhiar L, Dinh T, Schomacker KT, Panasyuk SV, Freeman JE, Lew R, et al. The use of medical hyperspectral technology to evaluate microcirculatory changes in diabetic foot ulcers and to predict clinical outcomes. Diabetes Care. 2007;30(4):903-10.

19. Chin JA, Wang EC, Kibbe MR. Evaluation of hyperspectral technology for assessing the presence and severity of peripheral artery disease. J Vasc Surg. 2011;54(6):1679-88.

20. Mayeur C, Campard S, Richard C, Teboul JL. Comparison of four different vascular occlusion tests for assessing reactive hyperemia using near-infrared spectroscopy. Crit Care Med. 2011;39(4):695-701.

21. Tseng SH, Bargo P, Durkin A, Kollias N. Chromophore concentrations, absorption and scattering properties of human skin in-vivo. Opt Express. 2009;17(17):14599-617.

22. Scheeren TW, Schober P, Schwarte LA. Monitoring tissue oxygenation by near infrared spectroscopy (NIRS): background and current applications. J Clin Monit Comput. 2012;26(4):279-87.

23. Fellahi JL, Butin G, Fischer MO, Zamparini G, Gerard JL, Hanouz JL. Dynamic evaluation of near-infrared peripheral oximetry in healthy volunteers: a comparison between INVOS and EQUANOX. J Crit Care. 2013;28(5):881.e1-6.

24. Steenhaut K, Lapage K, Bove T, De Hert S, Moerman A. Evaluation of different near-infrared spectroscopy technologies for assessment of tissue oxygen saturation during a vascular occlusion test. J Clin Monit Comput. 2016;31(6):1151-58.

25. Hyttel-Sorensen S, Hessel TW, Greisen G. Peripheral tissue oximetry: comparing three commercial near-infrared spectroscopy oximeters on the forearm. J Clin Monit Comput. 2014;28(2):149-55.

26. Samra SK, Stanley JC, Zelenock GB, Dorje P. An assessment of contributions made by extracranial tissues during cerebral oximetry. J Neurosurg Anesthesiol. 1999;11(1):1-5.

27. Creteur J, Carollo T, Soldati G, Buchele G, De Backer D, Vincent JL. The prognostic value of muscle $\mathrm{StO}_{2}$ in septic patients. Intensive Care Med. 2007;33(9):1549-56.

28. Futier E, Christophe S, Robin E, Petit A, Pereira B, Desbordes $\mathrm{J}$, et al. Use of near-infrared spectroscopy during a vascular occlusion test to assess the microcirculatory response during fluid challenge. Crit Care. 2011;15(5):R214.

29. Georger JF, Hamzaoui O, Chaari A, Maizel J, Richard C, Teboul JL. Restoring arterial pressure with norepinephrine improves muscle tissue oxygenation assessed by near-infrared spectroscopy in severely hypotensive septic patients. Intensive Care Med. 2010;36(11):1882-9.

30. Neviere R, Mathieu D, Chagnon JL, Lebleu N, Millien JP, Wattel F. Skeletal muscle microvascular blood flow and oxygen 
transport in patients with severe sepsis. Am J Respir Crit Care Med. 1996;153(1):191-5.

31. Gomez H, Torres A, Polanco P, Kim HK, Zenker S, Puyana JC, et al. Use of non-invasive NIRS during a vascular occlusion test to assess dynamic tissue $\mathrm{O}(2)$ saturation response. Intensive Care Med. 2008;34(9):1600-7.

32. Bezemer R, Lima A, Myers D, Klijn E, Heger M, Goedhart PT, et al. Assessment of tissue oxygen saturation during a vascular occlusion test using near-infrared spectroscopy: the role of probe spacing and measurement site studied in healthy volunteers. Crit Care. 2009;13(Suppl 5):S4.

Publisher's Note Springer Nature remains neutral with regard to jurisdictional claims in published maps and institutional affiliations. 